\title{
Obstacles and sources in dislocation dynamics: Strengthening and statistics of abrupt plastic events in nanopillar compression
}

\author{
S. Papanikolaou ${ }^{\mathrm{a}, *}$, H. Song ${ }^{\mathrm{a}, \mathrm{b}}$, E. Van der Giessen ${ }^{\mathrm{b}, *}$ \\ ${ }^{a}$ Department of Mechanical Engineering, The Johns Hopkins University, 3400 N Charles St, Baltimore, Maryland, 21218 \\ ${ }^{b}$ Zernike Institute for Advanced Materials, University of Groningen, 9747 AG Groningen, the Netherlands
}

\begin{abstract}
Mechanical deformation of nanopillars displays features that are distinctly different from the bulk behavior of single crystals: Yield strength increases with decreasing size and plastic deformation comes together with strain bursts or/and stress drops (depending on loading conditions) with a very strong sensitivity of the stochasticity character on material preparation and conditions. The character of the phenomenon is standing as a paradox: While these bursts resemble the universal, widely independent of material conditions, noise heard in bulk crystals using acoustic emission techniques, they strongly emerge primarily with decreasing size and increasing strength in nanopillars. In this paper, we present a realistic but minimal discrete dislocation plasticity model for the elasto-plastic deformation of nanopillars that is consistent with the main experimental observations of nano pillar compression experiments and provides a clear insight to this paradox. With increasing sample size, the model naturally transitions between the typical progressive behavior of nanopillars to a behavior that resembles evidence for bulk mesoscale plasticity. The combination of consistent strengthening, large flow stress fluctuations and critical avalanches is only observed in the depinning regime where obstacles are much stronger than dislocation sources; in contrast, when dislocation source strength becomes comparable to obstacle barriers, then yield strength size effects are absent but plasticity avalanche dynamics is strongly universal, across sample width and aspect-ratio scales. Finally, we elucidate the mechanism that leads to the connection between depinning and size effects in our model dislocation dynamics. In this way, our model builds a way towards unifying statistical aspects of mechanical deformation across scales.
\end{abstract}

Keywords: pillar compression, dislocation dynamics, size effect, abrupt plastic events, avalanches, depinning

\section{Introduction}

The dynamical character of crystal plasticity at the nanoscale has been under scrutiny for more than a decade (Uchic et al., 2003, 2004, 2005; Dimiduk et al., 2006; Uchic et al., 2009; Kraft et al., 2010; Greer and De Hosson, 2011). This interest is driven by the identification of unconventional plasticity size effects in the uniaxial deformation of samples made by the focused ion beam technique. Experiments of nanocrystalline pillar tension and compression have convincingly shown apparent strengthening with decreasing pillar width $w$, with the yield strength varying as $\sigma_{Y} \sim w^{-n}$ with $n \in(0.4,0.8)$ (Uchic et al., 2009; Greer and De Hosson, 2011), and a mild decrease with slenderness $\alpha=h / w$ (Volkert and Lilleodden, 2006; Kiener et al., 2008; Senger et al., 2011). The mechanism of strengthening in nanopillars has been attributed to the exhaustion of typical dislocation mechanisms and to a transition from typical Frank-Read sources in the bulk to the predominance of atypical sources such as surface sources (Gall et al., 2004; Park et al., 2006; Diao et al., 2006) and single-arm sources (Weinberger and Cai, 2008; Oh et al., 2009; Zheng et al., 2010; Lu et al., 2010; Ryu et al., 2015).

\footnotetext{
${ }^{*}$ Corresponding authors

Email address: spapanikolaou@jhu.edu, E.van.der.Giessen@rug.nl (E. Van der Giessen )
} 
Nano-strengthening is accompanied by large, abrupt strain jumps when the experiment is performed under load control) or by stress drops when under displacement control (Uchic et al., 2004; Dimiduk et al., 2005, 2006, 2007; Uchic et al., 2005; Greer et al., 2005; Greer and Nix, 2006; Ng and Ngan, 2007, 2008b,a; Shan et al., 2008). The stochastic abrupt events resemble noise/avalanches in disordered magnets or earthquakes (Weiss et al., 2000; Dimiduk et al., 2006; Uchic et al., 2005; Papanikolaou et al., 2012, 2011). Analysis of the statistics of abrupt plastic events has revealed that nanopillar events, statistically, appear to follow power-law-tailed distributions for strain steps with a large event cutoff that depends on specimen width (Weiss et al., 2000; Miguel et al., 2001a,b; Weiss and Marsan, 2003). The actual nature of these events, however has remained somewhat elusive, partially due to the complexity of loading paths, intertwining slip and stress events. In a complementary approach, acoustic emission (AE) measurements in a multitude of materials have revealed the presence of ubiquitous power-law plastic events that are independent of loading paths as well as of sample dimensions (Weiss et al., 2015; Miguel et al., 2001b). The energy release during such bulk events statistically displays a power law distribution with exponent $\tau \in(1.4,1.9)$ and no apparent cutoff dependence on sample parameters (Weiss et al., 2015); similarly, the exponent range in nanopillars is $\tau \in(1.3,2.1)$ for strain jumps or stress drops (depending on the selected loading path), but it shows strong fluctuations with dislocation density and sample dimensions (Greer and De Hosson, 2011).

While it may appear natural, it is not clear whether there is a causal relation between the nano-strengthening effects and the plastic avalanche statistics. Based on this premise, theoretical studies of the stochastic/abrupt plastic flow have mainly adopted continuum methods (Alava et al., 2014; Miguel et al., 2001b; Zaiser, 2006; Zaiser and Nikitas, 2007; Koslowski et at., 2004; Papanikolaou et al., 2012) thus addressing bulk properties, or two-dimensional discrete dislocation dynamics in the special environment of randomly placed edge dislocations in periodic systems with no obstacles or dislocation sources (Miguel et al., 2001a; Ispánovity et al., 2010, 2013; Alava et al., 2014). Such approaches have provided useful insights for predicting unconventional power-law statistics of abrupt plastic events in crystals (Weiss and Marsan, 2003), but they lack consideration of the mechanisms that give rise to nano-strengthening (starvation, source/obstacle exhaustion, single-arm source proliferation etc. ).

Dislocation sources can be either bulk -typically of the Frank-Read type- or unconventional, such as surface and single-arm sources; it is believed that bulk sources typically require much smaller average stress to be activated than surface ones (Greer and De Hosson, 2011). Dislocation obstacles capture the effect caused by precipitates, as well as forest dislocations that cross the glide slip planes. As molecular (MD) and three-dimensional discrete dislocation (3D-DDD) simulations (Madec et al., 2002) have shown, the strength of such obstacles varies strongly with dislocation configurations. Actual statistical properties of dislocation obstacle distributions currently remain unknown and thus, it is currently not feasible to investigate, using MD or 3D-DDD, the effect of obstacles on dislocation avalanche statistics. However, two-dimensional discrete edge dislocation (2D-DDD) simulations are ideal for the investigation of various obstacle-related statistical properties, since obstacles are minimally and randomly introduced with a pre-defined statistical distribution. Although the resulting statistical properties have not been explored in much detail, the interplay between dislocation sources and obstacles has been demonstrated in various 2D-DDD studies (e.g., Cleveringa et al., 2000; Balint et al., 2008; Deshpande et al., 2006; Chakravarty and Curtin, 2010). In fact, Chakravarty and Curtin (2010) have developed a direct connection between the yield strength of a system of edge dislocations in single slip and obstacle spacing, obstacle strength, source nucleation strength and average source spacing.

In this paper, we propose a minimal 2D discrete dislocation plasticity model for elastoplastic deformation of nanopillars and show that its predictions are are realistic in that they are consistent with the main experimental observations of strengthening as well as stochastic plastic flow. The explanation of the apparent paradox mentioned above naturally emerges through the stochastic competition among dislocation sources and obstacles.

The remainder of this paper is organized as follows: Section 2 describes the methodology of our 2D-DDD model that is designed to phenomenologically but minimally capture the details of nanopillar compression experiments by incorporating relatively strong surface and weak bulk sources. Also we present details of our statistical analysis of dislocation dynamics, similarly to common experimental protocols. Section 3 is focused on simulation results that are experimentally relevant, such as the behavior of the yield stress as a function of sample width and aspect ratio. In addition, we present the statistical aspect of pillar compression beyond yielding and we show 
qualitative agreement with uniaxial nanopillar compression experiments. Furthermore, we investigate the role of surface sources in our strengthening and statistics results, by performing extensive simulations in a simplified model where surface dislocation sources are absent, but the bulk properties remain unchanged. The relative role of source strength to obstacle strength in our simplified model is investigated in more detail in Section 4, showing that there are two qualitatively different regimes in terms of strengthening and statistics that we fully characterize. In Section 5, we summarize our results. In the Appendix, we show that our main conclusions do not alter when a second slip system is added.

\section{Model description and Methods}

The geometry of the model problem is shown in Fig. 1. Pillars are modeled by a rectangular profile of width $w$ and aspect ratio $\alpha(\alpha=h / w)$. Plastic flow occurs by the nucleation and glide of edge dislocations, for simplicity, on

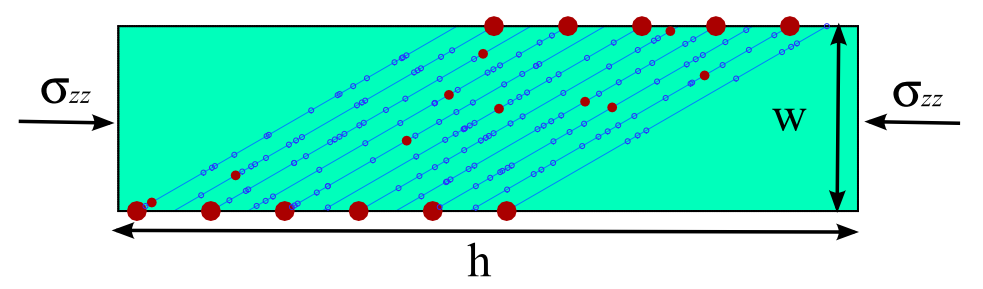

Figure 1: The 2D discrete dislocation plasticity model of pillar compression: Slip planes (lines) span the sample, equally spaced at $d=10 b$, where those close to corner slip plane are considered inactive to maintain a smooth loading boundary. Surface and bulk dislocation sources are present (red disks) and forest obstacles (small blue circles) are spread homogeneously across the active slip planes. Initially the sample is stress and dislocation free.

a single slip system. We confine attention to edge dislocations, not only because they provide the essential aspects of crystal plasticity (Burgers vector and line direction) with no numerical overhangs, but also because most results for collective plasticity avalanche dynamics originate from or are inspired by studies of edge dislocations (Miguel et al., 2001b; Ispánovity et al., 2010, 2013; Alava et al., 2014). With the typical Burgers vector of FCC crystals being $b=0.25 \mathrm{~nm}$, we study sample widths ranging in powers of 2 from $w=0.0625$ to $1 \mu \mathrm{m}$ with $\alpha=h / w=4$ to 32. The lateral edges $(x=0, w)$ are traction free, allowing dislocations to exit the sample. Loading is taken to be ideally strain-controlled, by prescribing the $z$-displacement at the ends of the pillar $(z=0, h)$. The applied strain rate, $\dot{h} / h=10^{4} \mathrm{~s}^{-1}$, is held constant across all simulations, similar to experimental practice.

Plastic deformation is described using the discrete dislocation framework for small strains (Van der Giessen and Needleman, 1995), where the determination of the state in the material employs superposition. As each dislocation is treated as a singularity in a linear elastic background solid with Young's modulus $E$ and Poisson ratio $v$, whose analytical solution is known at any position, this field needs to be corrected by a smooth image field $\left(^{\wedge}\right)$ to ensure that actual boundary conditions are satisfied. Hence, the displacements $u_{i}$, strains $\varepsilon_{i j}$, and stresses $\sigma_{i j}$ are written as

$$
u_{i}=\tilde{u}_{i}+\hat{u}_{i}, \varepsilon_{i j}=\tilde{\varepsilon}_{i j}+\hat{\varepsilon}_{i j}, \sigma_{i j}=\tilde{\sigma}_{i j}+\hat{\sigma}_{i j},
$$

where the ( ) field is the sum of the fields of all $N$ dislocations in their current positions, i.e.

$$
\tilde{u}_{i}=\sum_{J=1}^{N} \tilde{u}_{i}^{(J)}, \tilde{\varepsilon}_{i j}=\sum_{J=1}^{N} \tilde{\varepsilon}_{i j}^{(J)}, \tilde{\sigma}_{i j}=\sum_{J=1}^{N} \tilde{\sigma}_{i j}^{(J)} .
$$

Image fields are obtained by solving a linear elastic boundary value problem using finite elements with the boundary conditions changing as the dislocation structure evolves under the application of mechanical load.

The slip spacing in this paper is different from most 2D-DDD studies in the literature, where typically a larger slip spacing (such as $100 b$ or 200b) is used (e.g. Cleveringa et al., 2000; Deshpande et al., 2006; Chakravarty 
and Curtin, 2010). The possible consequence of such a large slip spacing relative to the Burgers vector is that interactions among neighboring slip planes are weakened. In our model, available (but not necessarily active) slip planes are $10 \mathrm{~b}$ apart and are oriented at $30^{\circ}$ away from the loading direction (Fig. 1). At the beginning of the calculation, the crystal is stress and dislocation free. This corresponds to a well-annealed sample, yet with pinned dislocation segments left that can act either as dislocation sources or as obstacles. Dislocations are generated from sources when the resolved shear stress $\tau$ at the source location is sufficiently high $\left(\tau_{\text {nuc }}\right)$ for a sufficiently long time $\left(t_{\text {nuc }}\right)$, mimicking the activation-expansion-closure process of a Frank-Read source. We consider bulk sources as in (Van der Giessen and Needleman, 1995) as well as surface sources, similar in spirit to (Nicola et al., 2007).

Each sample contains a random distribution of forest dislocation obstacles, surface dislocation sources, as well as a random distribution of bulk dislocation sources. The bulk sources are randomly distributed over slip planes at a density $\rho_{\text {nuc }}^{\text {bulk }}=60 \mu \mathrm{m}^{-2}$, while their strength is selected randomly from a Gaussian distribution with mean value $\bar{\tau}_{\text {nuc }}=50 \mathrm{MPa}$ and $10 \%$ standard deviation. Bulk sources are designed to mimic the Frank-Read mechanism in two dimensions, such that they generate a dipole of dislocations at distance $L_{\text {nuc }}$, when activated. The initial distance between the two dislocations in the dipole is

$$
L_{\mathrm{nuc}}=\frac{E}{4 \pi\left(1-v^{2}\right)} \frac{b}{\tau_{\mathrm{nuc}}},
$$

such that the shear stress of one dislocation acting on the other is balanced by the local shear stress. As we only consider glide of dislocations, their evolution is determined by the component of the Peach-Koehler force in the slip direction. For the Ith dislocation, this is given by

$$
f^{(I)}=\boldsymbol{n}^{(I)} \cdot\left(\hat{\boldsymbol{\sigma}}+\sum_{J \neq I} \tilde{\boldsymbol{\sigma}}^{(J)}\right) \cdot \boldsymbol{b}^{(I)},
$$

where $\boldsymbol{n}^{(I)}$ is the slip plane normal and $\boldsymbol{b}^{(I)}$ is the Burgers vector of dislocation $I$. This force will cause the dislocation $I$ to glide, following over-damped dynamics, with velocity

$$
v^{(I)}=\frac{f^{(I)}}{B},
$$

determined by the drag coefficient $B$. In this paper, its value is taken as $B=10^{-4} \mathrm{~Pa}$ s, which is representative for aluminum.

In addition to bulk dislocation sources, surface dislocation sources are successively placed at opposite ends of slip planes, which for the current slip plane spacing corresponds to a surface density of around $\rho_{\text {nuc }}^{\text {surf }}=175 / \mu \mathrm{m}$. We find that the surface source density dependence of our results is small for the widths and aspect ratios considered. Surface dislocation nucleation, through a sample surface instability (with no pinned segments), typically takes place at much higher stress than bulk dislocation multiplication, but the extent of possible mechanisms for dislocation nucleation from the surface is not yet clear, in contrast to typical bulk Frank-Read sources. 3D-DDD simulations (Ryu et al., 2015) have based the nucleation rate of surface sources on evidence from atomistic models: once surface nucleation happens (only allowed to occur on the $\frac{1}{2}\langle 110\rangle\{111\}$ type slip systems), the slip plane with the maximum Peach-Koehler force on the dislocation segment closest to the cylinder axis of the pillar is selected for dislocation nucleation, and then a half loop of radius $50 \mathrm{~b}$ is created and restricted to move inside the pillar. In our 2D model, we assume the probability of surface nucleation to depend on the assumed surface source strength; once the source is activated, a single dislocation is put at $10 \mathrm{~b}$ from the free surface, and will move according to the actual Peach-Koehler force. In the absence of interactions from any other dislocations, the Peach-Koehler force has to surpass the image stress of $312 \mathrm{MPa}$ when the dislocation is put at $10 \mathrm{~b}$ from the free surface, in order for the dislocation to move into the crystal rather than to escape through the free surface. Henceforth, our surface source has an effective nucleation strength of $312 \mathrm{MPa}$, approximately an order of magnitude larger than bulk sources. The applicability of this assumption will be verified in the subsequent sections by comparing our simulation results to typical experimental phenomenology. 
The density of bulk dislocation sources, $\rho_{\text {nuc }}^{\text {bulk }}=60 / \mu \mathrm{m}^{2}$, is chosen to be low enough such that the smallest sample width studied $(w=0.0625 \mu \mathrm{m})$ contains no bulk dislocation sources. This selection is consistent with the phenomenology of uniaxial nanopillar compression experiments, where it has been observed that dislocation starvation (no bulk dislocation sources) is present in samples with $w \lesssim 100 \mathrm{~nm}$ (Greer and De Hosson, 2011). In this limit, in our model, single dislocations are generated solely by surface sources with an effective nucleation strength of $312 \mathrm{MPa}$ that is higher than the average obstacle strength. Moreover, this surface dislocation nucleation strength is approximately an order of magnitude larger than the average dislocation source strength in the bulk at larger widths.

Once nucleated, dislocations can either exit the sample through the traction-free sides, annihilate with a dislocation of opposite sign when their mutual distance is less than $6 b$ or become pinned at an obstacle. Point obstacles are included to account for the effect of blocked slip caused by precipitates and forest dislocations on out-of-plane slip systems that are not explicitly described. They are randomly distributed over the slip planes with a constant density that corresponds on average, one source, either surface or bulk, that is accompanied by 8 randomly-distributed obstacles. The density of these obstacles amounts to approximately $5 \times 10^{10} / \mathrm{cm}^{2}$, which is consistent with the initial dislocation density in Ni nanopillars obtained by accurate measurements by Shan et al. (2008). It is worth mentioning that in this way the densities of sources and obstacles remains the same as the sample dimensions change, but there is a statistical preference towards always accompanying sources with obstacles in order to avoid statistical outlier behaviors. A dislocation stays pinned until its Peach-Koehler force exceeds the obstacle-dependent value $\tau_{\text {obs }} b$. The strength of the obstacles $\tau_{\text {obs }}$ is taken to be $300 \mathrm{MPa}$ with $20 \%$ standard deviation.

The simulation is carried out in an incremental manner, using a time step that is a factor 20 smaller than the nucleation time $t_{\text {nuc }}=10 \mathrm{~ns}$. At the beginning of every time increment, nucleation, annihilation, pinning at and release from obstacle sites are evaluated. After the dislocation structure is updated, the new stress field in the sample is determined, using the finite element method to solve for the image fields (Van der Giessen and Needleman, 1995).

Our simulations are carried out for material parameters that are reminiscent of aluminum: $E=70 \mathrm{GPa}, v=0.33$. We consider 50 random realizations of bulk sources and obstacles for each parameter case.

After plastic yielding, abrupt plastic events are defined as the sequence of consecutive stress drops $\delta \sigma-$ along the loading direction-, leading to the definition of equivalent energy release events by

$$
S=\frac{\sigma_{\mathrm{f}}}{4 E} \sum_{i \in \text { event steps }} \delta \sigma_{i}
$$

Here, $\sigma_{f}$ donates the flow stress after plastic yielding, computed by averaging the stress between $2 \%$ and $5 \%$ total strain. Defined this way, $S$ is connected for each sample to the energy release during a single avalanche across the plastic flow regime. Following Salerno and Robbins (2013); Zaiser and Nikitas (2007), possible avalanche behavior is detected through power law tails of the event probability distributions $P(S) \sim S^{-\tau} \mathcal{P}\left(S / S_{0}\right)$ with $S_{0}$ being the cutoff of the distribution.

\section{Strengthening size effects and statistical behavior of plasticity}

To investigate sample size effects, simulations were performed with different pillar widths $w$ ranging from $0.0625 \mu \mathrm{m}$ to $1 \mu \mathrm{m}$. The results reveal clear size effects in both the yield behavior and plastic flow regime. Fig. 2(a) shows typical examples of our predicted stress-strain curves, with characteristic strengthening and large flow stress fluctuations as $w$ decreases. Due to ideal strain-controlled loading, collective events emerge as stress drops. A typical slip pattern (for $w=1 \mu \mathrm{m}, \alpha=4$ ) that emerges, due to dislocation glide and escape from the free surface, is shown in Fig. 2(b).

\subsection{Yield stress and plastic flow stress fluctuations}

As shown in Fig. 3, we identify distinct size effects in the yield strength, defined similar to engineering practice as the average stress between $0.1 \%$ and $0.3 \%$ plastic strain. Fig. 3(a) shows that the yield strength $\sigma_{Y}$ decreases 


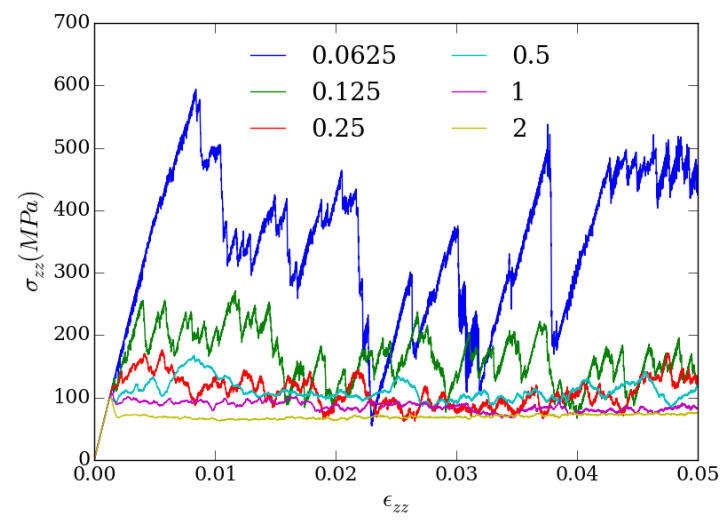

(a)

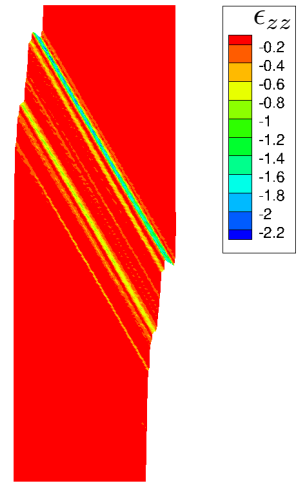

(b)

Figure 2: (a) Axial stress-strain curves, $\sigma_{z z}$ vs $\epsilon_{z z}$. Strengthening and large stress drops emerge as $w$ decreases, with the width shown in the legend, in $\mu \mathrm{m}$. (b) Uniaxial compression leads to characteristic slip patterns that are commonly observed in nanopillar uniaxial compression.

with increasing $w$. The fit to the aspect ratio $\alpha=4$ shows a clear power-law dependence $\sigma_{Y} \sim w^{-0.45}$ which is very close to experimental observations. In addition to a characteristic dependence on $w$, the sample strength depends on the aspect ratio $\alpha$, as also identified in recent experiments (Senger et al., 2011; Volkert and Lilleodden, 2006; Kiener et al., 2008). According to Fig. 3(b), the yield strength decreases strongly with a power law $\sigma_{Y} \sim \alpha^{-0.36}$ for small widths, while in larger samples, this dependence is virtually absent.

The yield strength also increases with the average dislocation density (Fig. 4(a) - right vertical axis) and its fluctuations (Fig. 4(a) - left vertical axis). The dislocation density fluctuations are defined through the standard deviation of the dislocation density $\left(\delta \rho=\int \sqrt{\rho(\epsilon)-\langle\rho>}\right) d \epsilon$ at the region around the yield stress, a quantity that demonstrates a strong variability even if the data points represent sample averages in 50 random realizations. The dislocation density and its standard deviation increase with sample strength and decrease with sample width (being proportional to the markersize), but these correlations do not emerge as clearly from our simulations because of unresolved sample-to-sample fluctuations. However, it is important to notice that this is the mobile dislocation density in our 2D-DDD simulations; the total dislocation density in 3D also contains off-plane dislocation segments,

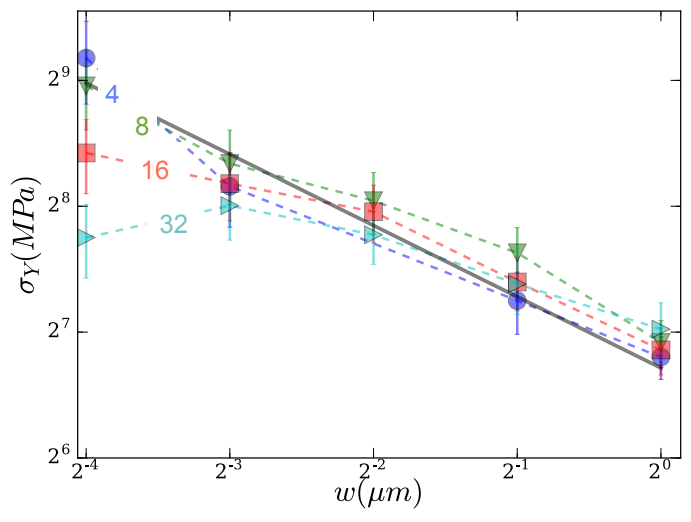

(a)

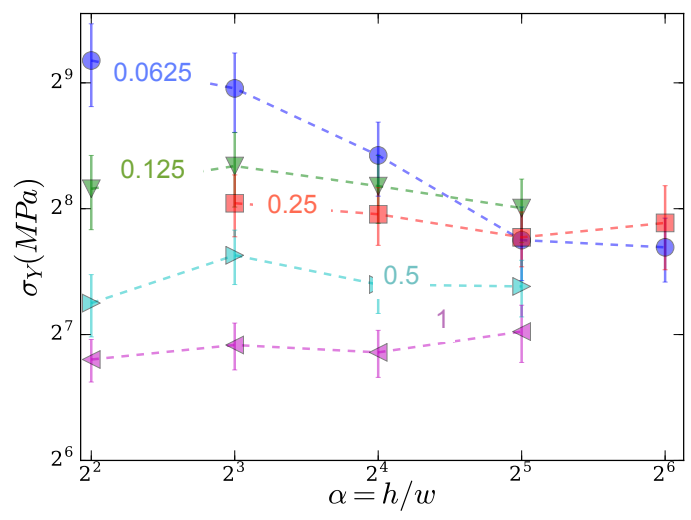

(b)

Figure 3: (a) The width dependence of the yield stress. Different aspect ratios $\alpha$ are indicated by colored numbers. The fit to $\alpha=4$ is shown in bold black line, (b) the dependence of the yield stress on the aspect ratio $\alpha$. 


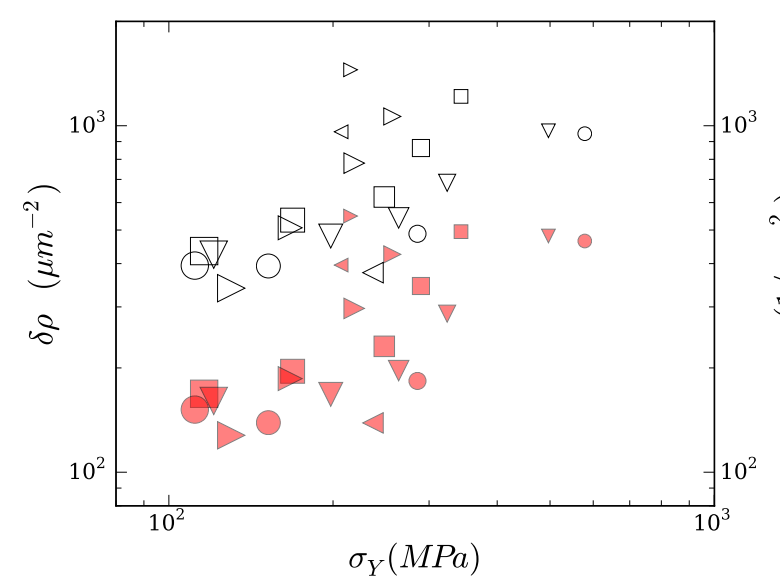

(a)

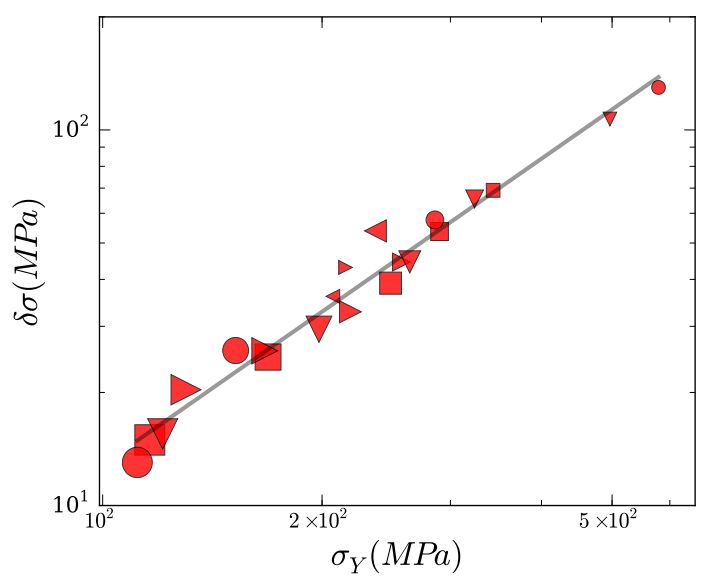

(b)

Figure 4: (a) Scaling of dislocation density and dislocation fluctuations with yield stress. The standard deviation (dark symbols) as well as the average (open symbols) of the dislocation density display an increasing trend with the yield stress. A number of aspect ratios are shown ( $\mathbf{\square}$ : $\alpha=4, \varangle: \alpha=8, \bullet: \alpha=16, \boldsymbol{\nabla}: \alpha=32, \varangle: 64$ ) with the symbol size proportional to the sample width: $w=0.0625,0.125,0.25,0.5,1.0 \mu$ m. (b) Flow stress fluctuations at $2 \%$ strain as a function of $\sigma_{Y}$, for the dependence studied in Fig. 3. Flow stress fluctuations at $2 \%$ strain as a function of $\sigma_{Y}$, for the dependencies studied in left panel( disks $\bullet: \alpha=4$, down-pointing triangles $\boldsymbol{\nabla}: \alpha=8$, squares $\boldsymbol{\square}: \alpha=16$, right-pointing triangles $\triangleright: \alpha=32$, with marker size denoting increasing $w$ ). Strong correlation with $\sigma_{Y}$ according to $\delta \sigma \sim \sigma_{Y}{ }^{1.84}$ (unbiased fit).

which are analogous to our forest obstacles.

Distinct from the yield strength, the flow stress $\sigma_{f}$ is defined as the average stress between $2 \%$ and $5 \%$ total strain. As shown in Fig. 4(b), flow stress fluctuations $\delta \sigma$ (defined as the standard deviation of $\sigma_{f}$ ) are strongly correlated to the yield strength with $\delta \sigma \sim \sigma_{Y}{ }^{1.84}$. While sample size influences the sample strength as expected, the observed correlation between stress fluctuation and stress is remarkable. Below we provide more insight into the connection between these fluctuations and the size dependence of the yield strength.

\subsection{Statistics of abrupt events}

Possible avalanche behavior is detected through power law tails of the event probability distributions $P(S) \sim$ $S^{-\tau} \mathcal{P}\left(S / S_{0}\right)$ with $S$ being defined in Eq. (6) and $S_{0}$ signifying the large size cutoff of the probability distribution (Salerno and Robbins, 2013; Zaiser and Nikitas, 2007). The onset of power-law behavior at decreasing width is clearly seen in Fig. 5a. Our findings are consistent with the existence of critical avalanche behavior $\left(S_{0} \rightarrow \infty\right)$ in the limit of infinitesimal width $w \rightarrow 0$ : This observation is justified by the fact that as $w \rightarrow 0$, obstacles in the active slip planes are at infinitesimal distance and therefore, pile-up behavior is not possible. In this limit, dislocation dynamics becomes fully characterized by the depinning behavior from existing obstacles. Further, the event distribution is characterized by an exponent $\tau=1.2 \pm 0.2$ (the line $P(S) \sim S^{-1.2}$ is shown as a guide to the eye) while $S_{0} \sim w^{-1}$. The existence of power-law behavior in the asymptotically small width limit becomes apparent in samples with low aspect ratio, as shown in the inset of Fig. 5a (the line for the average event size $S_{a v} \sim 1 / w$ is shown as a guide to the eye). In Fig. 5(b), $P(S)$ is shown for three different widths $(0.0625,0.25$ and $1 \mu \mathrm{m})$ and two aspect ratios (4 and 32). $P(S)$ displays power-law behavior across aspect ratios (the line $P(S) \sim S^{-1.2}$ is shown as a guide to the eye), when the width is $0.0625 \mu \mathrm{m}$; at larger widths, the distribution displays larger event sizes as the aspect ratio increases. This tendency is also seen in the behavior of $S_{a v}$ ( $c f$. inset of Fig. 5b), where independence of $\alpha$ is observed at small widths $(0.0625$ and $0.125 \mu \mathrm{m})$, while there is an increasing trend with $\alpha$, $S_{a v} \sim \alpha^{1}$, at large widths (with the line $S_{a v} \sim \alpha$ as a guide to the eye). Similar behavior is observed also in the dependence of the distribution cutoff $S_{0}$ as function of width and aspect ratio. The complete behavior of the abrupt event energy release distributions as function of aspect ratio, width and obstacle strength points towards dynamical critical behavior (Fisher, 1998) only in the limit of small widths and large aspect ratios. 


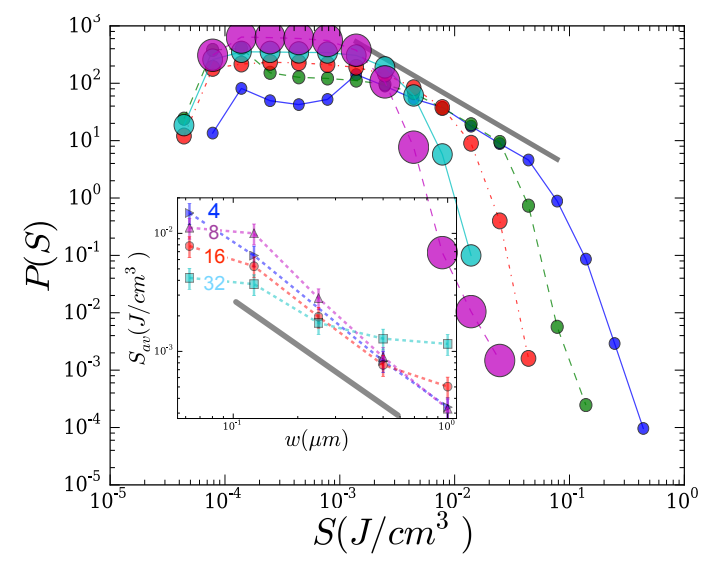

(a)

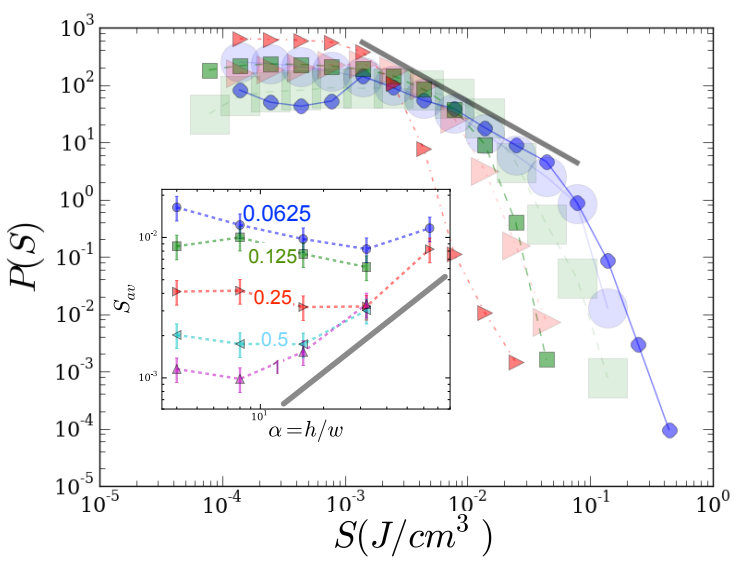

(b)

Figure 5: Histograms of abrupt events and cutoff dependence. (a) Width dependence of $P(S)$, demonstrating a clear power-law distribution as width decreases for $\alpha=4$ (here, symbol size reflects the width $w$ ). In the inset, the average event size is shown as a function of $w$ for different aspect ratios $\alpha$. (b) Dependence of abrupt event statistics on pillar aspect ratio $\alpha$. Three different widths $(\bullet: w=0.0625 \mu \mathrm{m}, \boldsymbol{\square}: w=0.25 \mu \mathrm{m}$, $\checkmark: w=1 \mu \mathrm{m}$ ) are shown for two different aspect ratios $\alpha=4$ and 32 (the symbol sizes follow the aspect ratio's magnitude for clarity).

\subsection{The role of surface dislocation sources for yield strength size effects and plastic burst distributions}

In our model, we hypothesize that surface dislocation sources capture the primary experimental phenomenology of nanopillar uniaxial compression by assuming that the surface sources have a much higher activation strength (312 MPa) than the bulk sources (50 MPa). Thus, the very existence of bulk sources naturally prevents surface sources from being activated. It is worth noting that molecular dynamics simulations support the existence of even higher activation barriers (of the order of 1GPa), as utilized in 3D-DDD simulations (Ryu et al., 2015). In practice, given the minimal character of our modeling, the phenomenology remains intact for any source strength larger than the average obstacle strength of $300 \mathrm{MPa}$.

It is natural to ask whether strengthening size effects and avalanche distributions are significantly affected by surface dislocation sources. Obviously, in our model, when $w=0.0625 \mu \mathrm{m}$, the role of surface dislocation sources is critical. However, is this true for $w>0.0625 \mu \mathrm{m}$ ? In order to answer this question, we perform simulations with all parameters intact as in the original model but with the surface dislocation sources removed. Then, our model contains only bulk sources and obstacles. In Fig. 6(a), if both surface and bulk dislocation sources and obstacles are present, the yield strength is overall highest since the number of total obstacles is largest (given that each surface source is accompanied by eight obstacles). By retaining the surface sources but removing the surface-related obstacles (blue line), the yield strength $\sigma_{Y}$ drops for all sizes, while the power-law dependence is approximately unchanged ( -0.5 compared to -0.45$)$. When, subsequently, also the surface dislocation sources are removed (red line), the yield strength $\sigma_{Y}$ remains the same on average for large $w$, because large samples have enough weak bulk sources that strong surface sources are not activated. However, as $w$ decreases, the exhaustion of bulk dislocation sources leads to a stronger strengthening size effect with power-law exponent -0.65 .

Just like for strengthening, the absence of surface sources and/or obstacles has an effect on avalanche distributions, but the difference in statistics has not been adequately resolved in our simulations, as is shown in Fig. 6(b). The statistics of the abrupt events for the small sample $w=0.125 \mu \mathrm{m}$ appears to point to similar behavior irrespective of the presence of surface dislocation sources. Furthermore, the effect of surface sources on statistics appears to be approximately absent with increasing sample size, as shown for $w=1 \mu \mathrm{m}$.

Until this point, all our simulations are characterized by weak dislocation sources and strong dislocation obstacles, and always the unstrained configuration is dislocation-free. Thus, it is natural to expect that when the sample size is large $(w \rightarrow \infty)$ the basic mechanism of yielding is pile-up dominated (cf. Chakravarty and Curtin, 2010). In this scenario, which we label as assisted dislocation depinning, a weak dislocation source nucleates enough 


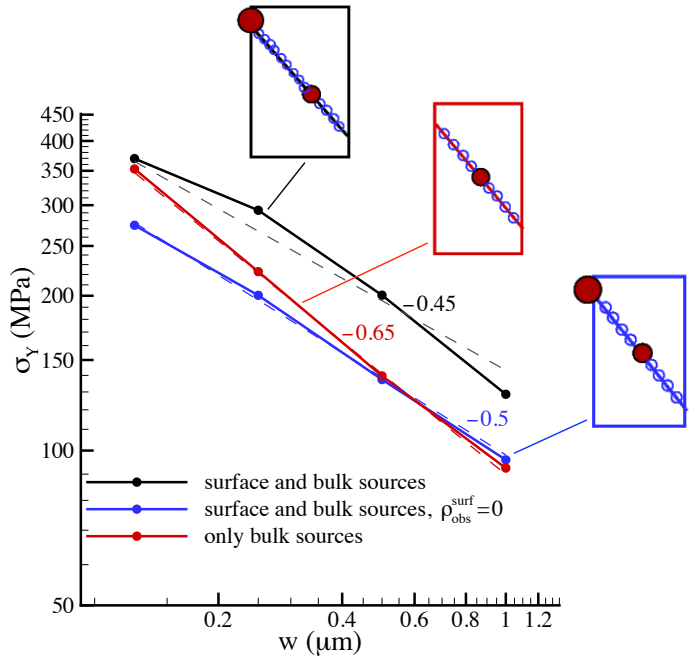

(a)

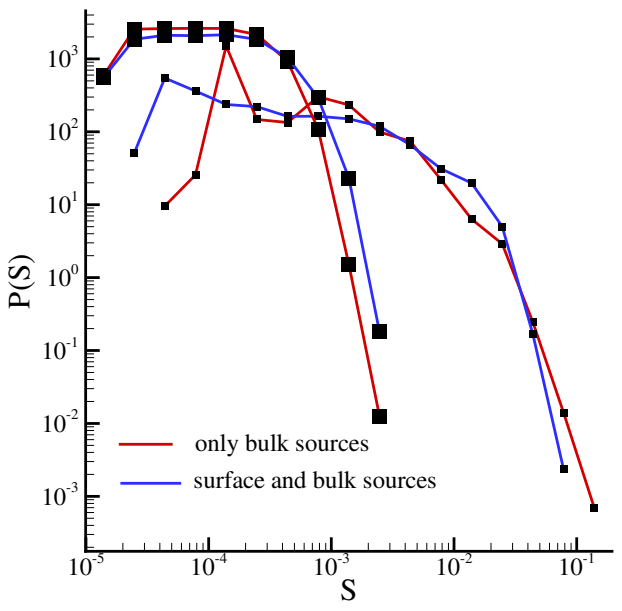

(b)

Figure 6: Comparison of results between with and without surface sources and surface related obstacles. (a) Yield stress dependence on $w$, (b) statistics (large $\mathbf{\square}: w=1 \mu \mathrm{m}$, small $\bullet: w=0.125 \mu \mathrm{m}$ ).

dislocations that can pile-up against an obstacle in order to form a large enough stress concentration that should move the leading dislocation across the obstacle. This mechanism is fully functional at large $w$, where the shear yield stress is close to the average dislocation nucleation resolved shear stress (50MPa) but much smaller than the average obstacle strength (300MPa). In the regime of assisted dislocation depinning, plastic flow is smooth and yielding is size-independent.

However, as the sample size decreases, assisted depinning is not anymore possible, since the space between nearby obstacles and sources decreases and therefore the available space in front of the obstacle is too small to generate a large enough pile-up stress. In this regime, dislocation pile-ups are "incomplete" and the yield strength increases to levels required for unassisted dislocation depinning of individual dislocations through obstacles. This mechanism resembles analogous elastic depinning phenomena through high-disorder landscapes (Fisher, 1998). Unassisted depinning naturally leads to strengthening, but also it leads to strongly abrupt and stochastic plastic flow, given that the stresses required for dislocation dynamics to be activated are highly correlated and stochastically defined. Thus, it is intuitively clear that unassisted dislocation depinning involves a deep, intrinsic connection between the yield strength size effect and the stochastic character of post-yield plastic flow.

Our main conclusion on the existence of this assisted/unassisted dislocation depinning is clearly associated with our choice of random distribution for dislocation sources and obstacles: Namely, as the sample size becomes smaller and therefore the number of sources in the bulk decreases, all bulk sources are on different active slip planes (since we consider closely spaced (10b) available slip planes). Then, we can keep the bulk obstacle density fixed through distributing the required number of obstacles on only the active slip planes. This is an appropriate statistical approach of sampling obstacles, for otherwise -if for example, obstacles are distributed randomly across all slip planes ( $c f$. Deshpande et al., 2006) - the behavior would be dominated by statistically outlier behaviors where slip planes exist with sources but without obstacles. In such a case, there is no dislocation pinning.

The assisted/unassisted dislocation depinning crossover takes place when dislocation sources activate at much lower stress than the stress required to surpass obstacles. In this limit, there is a simple but highly approximate estimate of the yield strength of an edge dislocation pile-up configuration on a single slip plane (Chakravarty and Curtin, 2010). Their estimate

$$
\sigma_{Y}=M^{-1} \sqrt{\tau_{\text {nuc }}^{2}+2 A b \tau_{\mathrm{obs}} / L_{\mathrm{obs}}}
$$




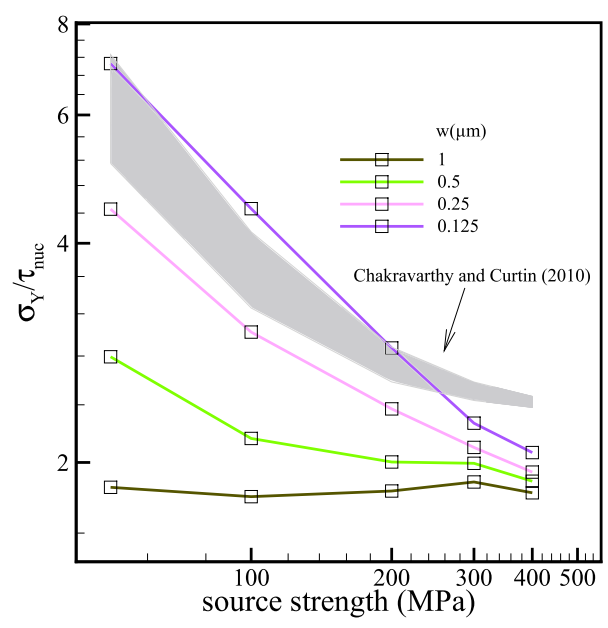

Figure 7: Comparison of simulations results with Chakravarty and Curtin (2010)'s estimate (7) where the map is plotted based on the variations ( \pm SDV) of $\tau_{\mathrm{obs}}$ and $\tau_{\text {nuc }}$ in our model.

expresses a strong dependence on obstacle spacing $L_{\mathrm{obs}}$ and the strength of obstacles, $\tau_{\mathrm{obs}}$ and of sources, $\tau_{\text {nuc }}(A$ is a fraction of the shear modulus, $M$ is the Schmid factor). As displayed in Fig. 7, our model predictions and this estimate come close only for small sample width with weak dislocation sources. The agreement in this limit $(w \rightarrow$ 0 ) is justified by the fact that the model of Chakravarty and Curtin (2010) does not account for interactions with dislocations on neighboring slip planes, something that is highly possible for large samples. In our model, when the sample width is small enough, active slip planes (at fixed dislocation source density) are at large mutual distance and therefore inter-slip interactions between dislocations become weak enough for single-slip-plane dislocation dynamics to dominate.

If the assisted/unassisted dislocation depinning crossover takes place in the weak-source (compared to obstacles) limit, it is natural to question what happens when source activation and obstacle strength have a similar value. The possibility of similar obstacle and source strengths is guaranteed by the strong variability of obstacle strengths due to randomness in the initial dislocation configuration and the random character of dislocation forest obstacles. This range in parameter space will be explored in the next session.

\section{Source $v s$. obstacle strength and the role for yield strength, size effects and statistical behavior}

We present the results of extensive simulations to explore the dependence of the strengthening and statistics on the ratio of dislocation source to obstacle strength, $\tau_{\text {nuc }} / \tau_{\text {obs }}$. The obstacle strength is kept unchanged $\left(\tau_{\mathrm{obs}}=300\right.$ MPa with $20 \%$ SDV), while the source strength $\tau_{\text {nuc }}$ is varied from $50 \mathrm{MPa}$ to $400 \mathrm{MPa}$. As may be seen in Fig. 8(a), an increase of $\tau_{\text {nuc }} / \tau_{\text {obs }}$ leads to size-independent yield strength. This is a concrete signature that the yield strength size effect is strongly controlled by the assisted/unassisted dlslocation depinning mechanism we proposed.

Obstacle strength as well as obstacle density influence dislocation plasticity. In particular, the yield strength has a strong dependence on the density of obstacles: more obstacles results in more probable unassisted depinning. Furthermore, it appears that the obstacle density is the key factor ( $c f$. Fig. 8(b)) that determines the yield strength: for small widths, the increase of obstacle density leads to a large increase of the yield stress in small samples. At large widths, the increase of obstacle density does not strongly influence the strength, as expected for a system that forms large dislocation pile-ups. 
The role of obstacles is conditionally defined relative to the activity of dislocation sources. Our original model was defined in Section 2 to compare well to experimental phenomenology of uniaxial nanopillar compression (see Section 3) by assuming that bulk dislocation sources have a much lower activation stress than the strength of dislocation obstacles. In this way, the change of width $w$ leads to a crossover between assisted (at large $w$ ) -where pile-up behavior can take place- and unassisted (at small $w$ ) dislocation depinning behavior, before dislocation starvation takes place at the ultra-small pillar regime. However, it is clear that there is another fundamentally different regime of dislocation dynamics, namely when the source strength becomes comparable to the obstacle strength. In the regime where bulk sources are as strong as the dislocation obstacles, yield strength size effects are not present anymore ( $c f$. Fig. 8(a)), but what about the corresponding statistical/stochastic behavior of the events?

The statistics of plastic events is shown in Fig. 9 for the two cases of dislocation sources with small vs. large activation strengths, keeping the obstacle strength fixed. When sources are weaker than the obstacles (cf. Fig. 9(a)), the overall dependence on sample width $w$ is analogous to our main model and the results discussed in Section 2, with a large sensitivity of the distribution cutoff on the sample width. When source strength becomes comparable to obstacle strength, the statistics changes dramatically becoming approximately a pure power-law behavior, with a large-event cutoff that seems to be independent of sample dimensions and geometry. Therefore, the statistical behavior of abrupt plastic events evidently appears universal across widths and aspect ratios (cf. Fig. 9(b)). This drastically new regime of source-driven deformation contains statistical noise that resembles acoustic emission measurements of crystalline materials (Weiss et al., 2000; Weiss and Marsan, 2003; Weiss et al., 2015).

The drastically different character of plastic events can also be traced in the spatial and temporal character of the dislocation motion during the energy release events. As shown in Fig. 10, weaker sources lead to large, long-range inter-slip correlations (seen by the lateral strain correlations at large strains) - facilitating avalanche events. Nevertheless, these events involve only a few dislocations at a time, consistent with experiments and other dislocation dynamics simulations (Csikor et at., 2007; El-Awady, 2015). In contrast, when the source strength is comparable to the obstacle strength, events are traced in dominant dislocation pile-ups on a single slip plane. Long pile-ups accumulated during plastic flow generate long-range stress fields that lead to activation of nearby bulk dislocation sources. It is noteworthy that dislocation interactions lead to the fact that regions of strain localization are associated with a high dislocation density, as shown in Fig. 11. Dislocation dynamics is dominated by the

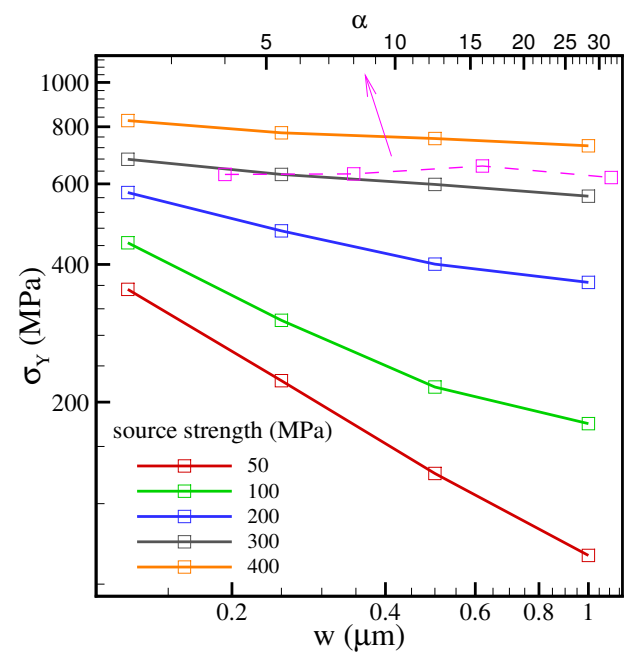

(a)

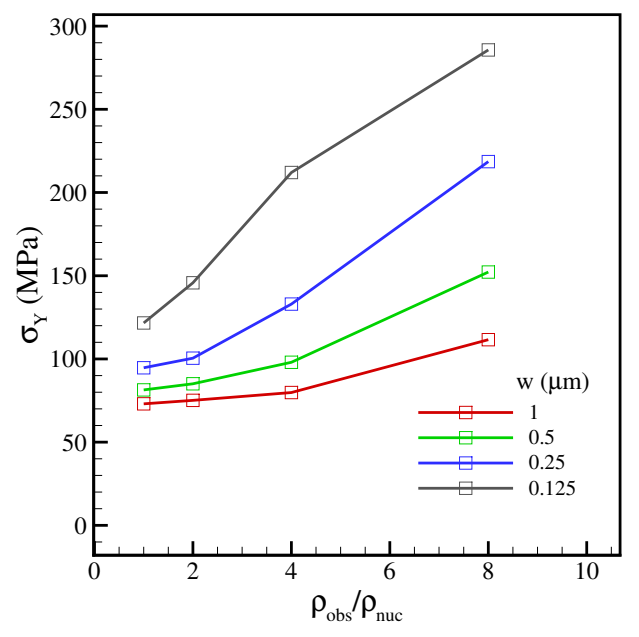

(b)

Figure 8: (a) Dependence of strengthening on the source strength. Different aspect ratio is shown for $w=0.25 \mu \mathrm{m}$ when source strength is 300 $\mathrm{MPa}$, (b) Dependence of $\sigma_{\mathrm{Y}}$ on the obstacle-to-bulk source density for different sample width. 


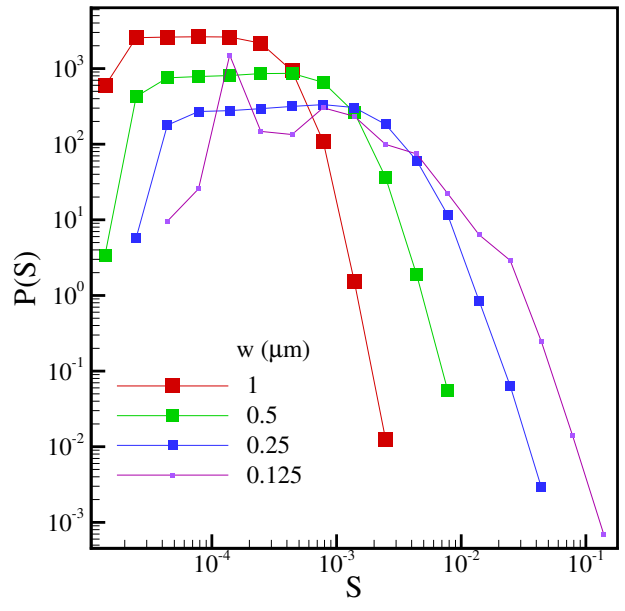

(a)

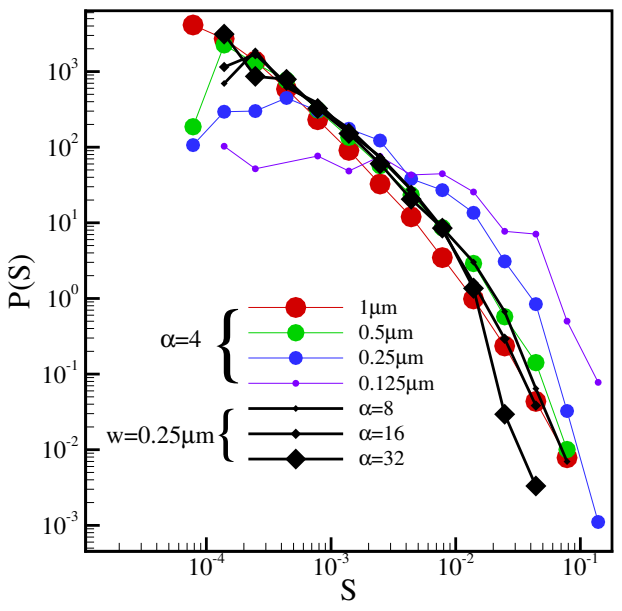

(b)

Figure 9: Events statistics for different source strength $\tau_{\text {nuc }}$ (obstacle strength $\tau_{\text {obs }}=300 \mathrm{MPa}$ with $20 \%$ SDV). The size of the symbol stands for $w$ from $1 \mu \mathrm{m}$ (largest symbol) to $0.125 \mu \mathrm{m}$ (smallest symbol). (a) When $\tau_{\text {nuc }}=50 \mathrm{MPa}$, (b) when $\tau_{\text {nuc }}=300 \mathrm{MPa}$, blue lines are the statistics for $w=0.25 \mu \mathrm{m}$ with different aspect ratios, symbol size stands for the value of aspect ratio.

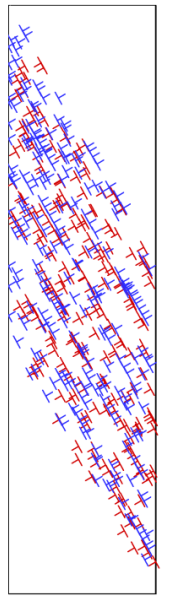

(a)

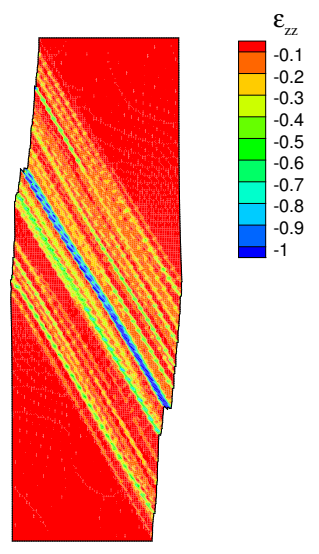

(b)

Figure 10: (a) Dislocation configuration (plotted in undeformed configuration), and (b) distribution of strain along the loading direction (plotted in deformed configuration) in a sample of $w=1 \mu \mathrm{m}, \alpha=4$ with an average source strength of $50 \mathrm{MPa}$ at $5 \%$ strain.

yielding of a primary slip band with high dislocation density and large frustration between oppositely signed dislocations. This can be seen, for example, in Fig. 11 where most activity originates in the large dislocation density at the slip planes near the shear band, dominated by both dislocation signs on nearby but on different slip planes. This is a novel regime of dislocation friction in dislocation dynamics, where the frictional "contacts" are formed by high concentrations of oppositely-signed dislocations. The result is a self-organized dynamical critical behavior, analogous to self-organized earthquake faults (Fisher, 1998): the motion of a few dislocations on the predominant slip plane drags along a large collection of nearby dislocations. This structural behavior is 
reminiscent of the coarse-slip-band (CSB) phenomenon (Asaro and Rice, 1977) which is believed to lie at the core of crystal plasticity instabilities (Dao and Asaro, 1996). This behavior should be contrasted to the more homogeneous deformation in the case of weak sources ( $c f$. Fig. 10).

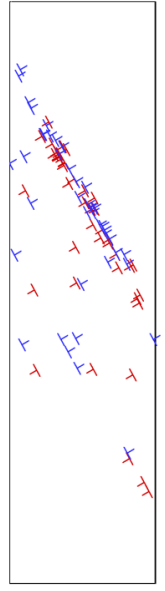

(a)

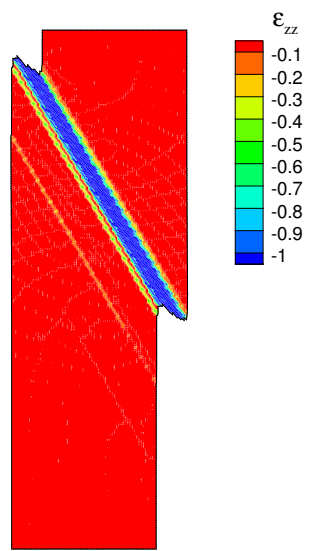

(b)

Figure 11: (a) Dislocation configuration strain (plotted in undeformed configuration), and (b) distribution of strain along the loading direction (plotted in deformed configuration) in a sample of $w=1 \mu \mathrm{m}, \alpha=4$ with an average source strength of $300 \mathrm{MPa}$ at $5 \%$ strain.

\section{Summary}

In this work, we presented a minimal model that captures the basic aspects of uniaxial compression of nanopillars, including yield strength size effects as well as stochastic effects of post-yield plastic flow. We have identified that the critical ingredients for such predictions are: a higher density of obstacles than dislocation sources in the bulk; the presence of dislocation sources on the surface that are stronger than those in the bulk; and, a spacing between potentially active slip planes of tens of Burgers vectors. It bears emphasis that the dislocation sources and obstacles in our model follow a stochastic Gaussian distribution with $10 \%$ relative fluctuations. Analogous relative fluctuations should be expected in realistic microstructures, but the Gaussian character of these distributions in nanopillars may be an assumption that should be revisited in future studies.

When sources are, on average, much weaker than obstacles, strengthening with decreasing width is consistent with the experimentally observed scaling $\sigma_{Y} \sim w^{-0.45}$. Energy release events statistically become larger as width decreases, and the statistical distribution of events $P(S)$ acquires a power-law tail in the limit of small widths with an exponent $\tau=1.5 \pm 0.2$ in $P(S) \sim S^{-\tau}$. When dislocation sources are comparable in strength or stronger than the obstacles, the strength is virtually independent of width or aspect ratio while the statistical distribution of plastic events appears universal across width and aspect ratio, scaling with $\tau=1.9 \pm 0.2$.

We demonstrated that small activation strengths (compared to obstacle strengths) for dislocation sources leads to assisted dislocation depinning at large widths that is strongly associated with dislocation pile-ups, while the behavior switches to an unassisted dislocation depinning mechanism at small widths, where dislocations jump over obstacles individually without being assisted by dislocation pile-ups. The crossover that we identified naturally leads to strengthening that is consistent with the experimental phenomenology of BCC/FCC uniaxial nanopillar compression, but also it leads to the onset of critical avalanches in the limit of small widths, where obstacles on active slip planes are asymptotically close. Stochastic plastic flow fluctuations are strongly connected to the yield strength through an almost quadratic dependence $\delta \sigma_{f} \sim \sigma_{Y}^{1.84}$, a prediction that is deeply rooted in the nature of our unassisted dislocation depinning mechanism. This mechanism clearly distinguishes nanopillar crystals from 
other materials which display abrupt plastic flow, such as bulk metallic glasses (Zhang et al., 2005), but with no such correlations.

Furthermore, we demonstrated that high activation strengths for dislocation sources, and given that the unstrained samples are mechanically annealed, lead to the absence of size effects and universal plastic flow across sample widths and aspect ratios. It is important to note that this size independent regime for strong dislocation sources should exist for any dislocation source density because of the fact that the weakest of these very strong sources will dominate the plastic behavior of the sample (irrespective of their location). We identified the reason of this behavior to originate in the onset of large dislocation frustration along a predominant slip band in the sample. Such crackling slip bands resemble the onset of coarse shear bands (CSBs) in crystal plasticity.

\section{Acknowledgments}

We would like to thank D.M. Dimiduk, C.F. Woodward, E. Lilleoden, S. Zapperi and P. Ispanovity for inspiring comments and discussions. This work has been supported through a VIDI Grant (NWO, SP) as well as a DOEBES grant (SP) and a FOM grant (HS, EVdG). This work benefited greatly from the facilities and staff of the High Performance Computing Center at the Johns Hopkins University as well as the Zernike Institute for Advanced Materials at The University of Groningen, the Netherlands.

\section{References}

Alava, M.J., Laurson, L., Zapperi, S., 2014. Crackling noise in plasticity. Eur. Phys. J. Spec. Top. 223, 2353.

Asaro, R.J., Rice, J.R., 1977. Strain Localization in Ductile Single Crystals. J. Mech. Phys. Solids 25, 309.

Balint, D.S., Deshpande, V.S., Needleman, A., Van der Giessen, E., 2007. Discrete dislocation plasticity analysis of the grain size dependence of the flow strength of polycrystals. Int. J. Plast. 24, 2149-2172.

Chakravarty, S.S., Curtin, W.A., 2010. Effect of source and obstacle strengths on yield stress: a discrete dislocation study. J. Mech. Phys. Solids $58,625-635$.

Cleveringa, H.M.M., Van der Giessen, E., Needleman, A., 1999. A discrete dislocation analysis of bending. Int. J. Plast. 15, 837-868.

Csikor, F.F., Motz, C., Weygand, D., Zaiser, M., Zapperi, S, 2007. Dislocation Avalanches, Strain Bursts, and the Problem of Plastic Forming at the Micrometer Scale. Science 318, 251.

Diao, J.K., Gall, K., Dunn, M.L., Zimmerman, J.A., 2006. Atomistic simulations of the yielding of gold nanowires. Acta Mater. 54, 643-653.

Dao, M., Asaro, R.J., 1996. Localized Deformation Modes and non-Schmid Effects in Crystalline Solids, Part I: Critical Conditions for Localization. Mech. Mater. 23, 71.

Deshpande, V.S., Needleman, A., Van der Giessen, E., 2005. Plasticity size effects in tension and compression of single crystals. J. Mech. Phys. Solids 53, 2661-2691

Dimiduk, D.M., Uchic, M.D., Parthasarathy, T.A., 2005. Size-affected single-slip behavior of pure nickel microcrystals. Acta Mater. 53, 4065.

Dimiduk, D.M., Woodward, C.F., LeSar, R., Uchic, M.D., 2006. Scale-free intermittent flow in crystal plasticity. Science 312, 1188.

Dimiduk, D.M., Uchic, M.D., Rao, S.I., Woodward, C.F., Parthasarathy, T.A., 2007. Overview of experiments on microcrystal plasticity in FCC-derivative materials: selected challenges for modelling and simulation of plasticity. Model. Simulat. Mater. Sci. Eng. 15, 135.

El-Awady, J., 2014. Unravelling the physics of size-dependent dislocation-mediated plasticity. Nat. Comm. 6, 5926.

Fisher, D.S., 1998. Collective transport in random media: from superconductors to earthquakes. Phys. Rep. 301, 113-150.

Gall, K., Diao, J.K., Dunn, M.L., 2004. The strength of gold nanowires. Nano Letters 4, 2431-2436.

Greer, J.R., De Hosson, J.T.M., 2011. Plasticity in small-sized metallic systems: Intrinsic versus extrinsic size effect. Progress in Materials Science 56, 654 .

Greer, J.R., Nix, W.D., 2006. Nanoscale gold pillars strengthened through dislocation starvation. Phys. Rev. B 73, 245410

Greer, J.R., Oliver, W.C., Nix, W.D., 2005. Size dependence of mechanical properties of gold at the micron scale in the absence of strain gradients. Acta Mater. 53, 1821.

Ispánovity, P.D., Groma, I., Györgyi, G., Csikor, F.F., Weygand, D., 2010. Submicron plasticity: yield stress, dislocation avalanches, and velocity distribution. Phys. Rev. Lett. 105, 085503.

Ispánovity, P.D., Hegyi, Á., Groma, I., Györgyi, G., Ratter, K., Weygand, D., 2013. Average yielding and weakest link statistics in micron-scale plasticity. Acta Mater. 61, 6234.

Kiener, D., Grosinger, W., Dehm, G., Pippan, R., 2008. A further step towards an understanding of size-dependent crystal plasticity: In situ tension experiments of miniaturized single-crystal copper samples. Acta Mater. 56, 580.

Koslowski, M., LeSar, R., Thomson, R., 2004. Avalanches and Scaling in Plastic Deformation. Phys. Rev. Lett. 93, 125502.

Kraft, O., Gruber, P. A., Mönig, R., Weygand, D., 2010. Plasticity in confined dimensions. Ann. Rev. Mater. Res. 40, 293.

Lu, Y., Huang, J.Y., Wang, C., Sun, S.H., Lou, J., 2010. Cold welding of ultrathin gold nanowires. Nat. Nanotechnol. 5, 218-224.

Madec, R., Devincre, B., Kubin, L.P., 2002. From dislocation junctions to forest hardening. Phys. Rev. Lett. 89, 255508. 
Miguel, M., Vespignani, A., Zapperi, S., Weiss, J., Grasso, J.R., 2001. Complexity in dislocation dynamics: model. Mater. Sci. Eng. A 30(9), 324-327.

Miguel, M.-C., Vespignani, A., Zapperi, S., Weiss, J., Grasso, J.R., 2001. Intermittent dislocation flow in viscoplastic deformation. Nature 410 (2001), 667.

Ng, K.S., Ngan, A.H.W., 2007. Creep of micron-sized aluminium columns, Phil. Mag. Lett.,87, 967.

Ng, K.S., Ngan, A.H.W., 2008a. Stochastic nature of plasticity of aluminum micro-pillars, Acta Mater. 56, 1712.

Ng, K.S., Ngan, A.H.W., 2008b. A Monte Carlo model for the intermittent plasticity of micro-pillars. Model. Simulat. Mater. Sci. Eng. 16, 055004.

Nicola, L., Bower, A.F., Kim, K.-S., Needleman, A., Van der Giessen, E., 2007. Surface versus bulk nucleation of dislocations during contact. J. Mech. Phys. Solids 55, 1120-1144.

Oh, S.H., Legros, M., Kiener, D. Dehm, G., 2009. In situ observation of dislocation nucleation and escape in a submicrometre aluminium single crystal. Nat. Mater. 8(2), 95-100.

Papanikolaou, S., Bohn, F., Durin, G., Sommer, R.L., Zapperi, S., Sethna, J.P., 2011. Universality beyond power laws and the average avalanche shape, Nat. Phys. 7, 316.

Papanikolaou, S., Dimiduk, D.M., Choi, W., Sethna, J.P., Uchic, M.D., Woodward, C.F., Zapperi, S., 2012. Quasi-periodic events in crystal plasticity and the self-organized avalanche oscillator. Nature, 490, 517-521.

Park, H.S., Gall, K., Zimmerman, J.A., 2006. Deformation of FCC nanowires by twinning and slip. J. Mech. Phys. Solids 54, $1862-1881$.

Ryu, I., Cai, W., Nix, W.D., Gao, H., 2015. Stochastic behaviors in plastic deformation of face-centered cubic micropillars governed by surface nucleation and truncated source operation. Acta Mater. 95, 176-183.

Salerno, K.M., Robbins, M.O., 2013. Effect of inertia on sheared disordered solids: Critical scaling of avalanches in two and three dimensions. Phys. Rev. E 88, 062206.

Senger, J., Weygand, D., Motz, C., Gumbsch, P., Kraft, O., 2011. Aspect ratio and stochastic effects in the plasticity of uniformly loaded micrometer-sized specimens. Acta Mater., 59, 2937.

Shan, Z.W., Mishra, R., Asif, S.A.S., Warren, O.L., Minor, A.M., 2008. Mechanical annealing and source-limited deformation in submicrometre-diameter Ni crystals, Nat. Mater. 7, 115.

Uchic, M.D., Dimiduk, D.M., Florando, J.N., Nix, W.D., 2003. Exploring specimen size effects in plastic deformation of Ni3(Al, Ta). Mater. Res. Soc. Symp. Proc. 753: BB1-4.

Uchic, M.D., Dimiduk, D.M., Florando, J.N., Nix, W.D., 2004. Sample dimensions influence strength and crystal plasticity. Science $305,986$.

Uchic, M.D., Dimiduk, D.M., 2005. A methodology to investigate size scale effects in crystalline plasticity using uniaxial compression testing. Mater. Sci. Eng. A400, 268-278.

Uchic, M.D., Shade, P. A., Dimiduk, D.M., 2009. Plasticity of micrometer-scale single crystals in compression. Annu. Rev. Mater. Sci. 39, 361.

Van der Giessen, E., Needleman, A., 1995. Discrete dislocation plasticity: a simple planar model. Model. Simul. Mater. Sci. Eng. 3, 689.

Volkert, C.A., Lilleodden, E.T., 2006. Size effects in the deformation of sub-micron Au columns. Phil. Mag. 86, 5567-5579.

Weinberger, C.R., Cai, W., 2008. Surface-controlled dislocation multiplication in metal micropillars. Proc. Natl. Acad. Sci. 105(38), 1430414307.

Weiss, J., Lahaie, F., Grasso, J.R., 2000. Statistical analysis of dislocation dynamics during viscoplastic deformation from acoustic emission, J. Geophys. Res. 105, 433.

Weiss, J., Marsan, D., 2003. Three-dimensional mapping of dislocation avalanches: clustering and space/time coupling. Science 89, 299.

Weiss, J., Rhouma, W.B., Richeton, T., Dechanel, S., Louchet, F., Truskinovsky, L., 2015. From Mild to Wild Fluctuations in Crystal Plasticity. Phys. Rev. Lett. 114, 105504.

Zaiser, M., 2006. Scale invariance in plastic flow of crystalline solids. Adv. Phys. 55, 185-245.

Zaiser, M., Nikitas, N., 2007. Slip avalanches in crystal plasticity: scaling of the avalanche cut-off. J. Stat. Mech. Theor. Exp. 2007 , P04013.

Zhang, Z.F., Zhang, H., Pan, X.F., Das, J., Eckert, J., 2005. Effect of aspect ratio on the compressive deformation and fracture behaviour of Zr-based bulk metallic glass. Phil. Mag. Lett. 85, 513.

Zheng, H., Cao, A., Weinberger, C.R., Huang, J.Y., 2010. Discrete plasticity in sub-10-nm-sized gold crystals. Nat. Comm. 1, 144.

\section{Appendix}

In the main text, we present results for only one slip system, $c f$. Fig. 1. Here, we verify strengthening (cf. Fig. 3(a)) as well events statistics ( $c f$. Fig. 5(a)) when a second slip system is present, oriented for symmetric double slip under the applied uniaxial straining. Fig. 12 reveals that the key features that were discussed in the main text for single slip persist, such as the experimentally comparable yield strength size effect and the strong sensitivity of large event cutoff on sample width. Given that the number of surface dislocation sources is necessarily doubled, the inclusion of 50 samples appears to not completely resolve the strong statistical fluctuations in the distributions. However, given that the primary features of our work remain intact for the double-slip system, we focus solely on the single-slip system for computational convenience. 


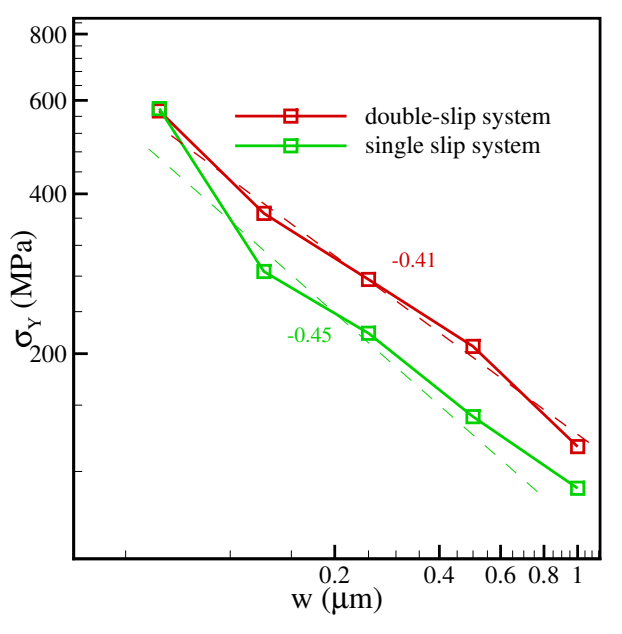

(a)

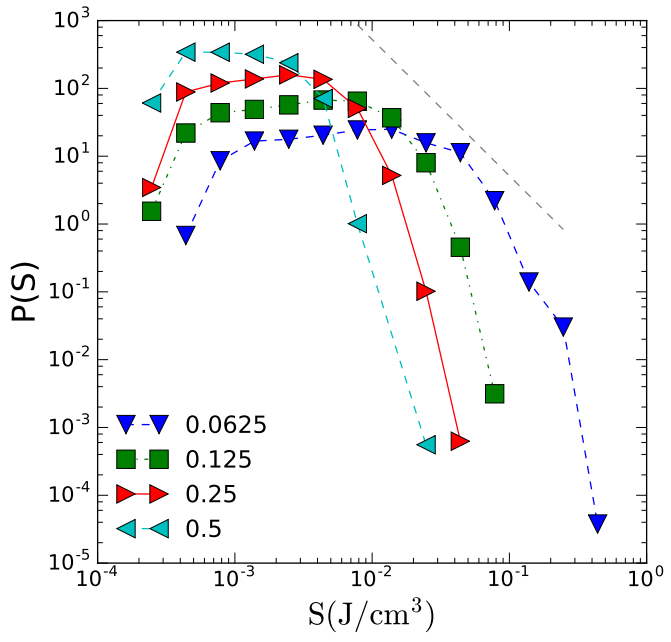

(b)

Figure 12: (a) Dependence of strengthening on $w$ in the case of two slip systems. Dashed lines signify guides to the eye for power law strengthening with exponent labeled on the figure. (b) Associated statistics when $w$ changes from $1 \mu \mathrm{m}$ (purple) to $0.0625 \mu \mathrm{m}$ (blue). The dashed line signifies an inverse-quadratic power-law and it just serves as a guide to the eye. 